\title{
Stochastic Taylor expansions for the expectation of functionals of diffusion processes
}

\author{
Andreas Rößler \\ Darmstadt University of Technology, Fachbereich Mathematik, Schlossgartenstr.7, \\ D-64289 Darmstadt, Germany
}

\begin{abstract}
Stochastic Taylor expansions of the expectation of functionals applied to diffusion processes which are solutions of stochastic differential equation systems are introduced. Taylor formulas w.r.t. increments of the time are presented for both, Itô and Stratonovich stochastic differential equation systems with multi-dimensional Wiener processes. Due to the very complex formulas arising for higher order expansions, an advantageous graphical representation by coloured trees is developed. The convergence of truncated formulas is analyzed and estimates for the truncation error are calculated. Finally, the stochastic Taylor formulas based on coloured trees turn out to be a generalization of the deterministic Taylor formulas using plain trees as recommended by Butcher for the solutions of ordinary differential equations.
\end{abstract}

Key words: stochastic Taylor expansion, stochastic differential equation, diffusion process

MSC 2000: 60H10, 65C30, 60J60, 41A58

\section{Introduction}

Taylor expansions are well known in the deterministic setting. They play an important role, e.g. for the determination of numerical schemes for the approximation of solutions of differential equations. Especially the modern theory of order conditions for Runge-Kutta methods due to Butcher [4] is based upon expansions w.r.t. trees. A generalization of the deterministic Taylor formula for a class of Itô processes has been introduced by Platen and Wagner [10].

Email address: roessler@mathematik.tu-darmstadt.de (Andreas Rößler). 
They make use of hierarchical sets in order to specify the Taylor expansion of a functional $f$ of the solution process $X=\left(X_{t}\right)_{t \geq t_{0}}$ of a stochastic differential equation (SDE) (see also [8]). In contrast to the expansion of $f\left(X_{t}\right)$, a Taylor expansion of the expectation $E\left(f\left(X_{t}\right)\right)$ is presented in the following. Instead of using hierarchical sets, coloured rooted trees are used for the calculation of the Taylor expansion. For example, a representation with the aid of coloured trees is the key to the stochastic numerical analysis, especially to the determination of Runge-Kutta methods for SDEs as investigated for strong approximation by Burrage and Burrage [2,3] and for weak approximation by Rößler [11].

Let $(\Omega, \mathcal{A}, P)$ be a probability space with a filtration $\left(\mathcal{F}_{t}\right)_{t \geq 0}$ and let $I=\left[t_{0}, T\right]$ for some $0 \leq t_{0}<T<\infty$. We consider the solution $\left(X_{t}\right)_{t \in I}$ of a $d$-dimensional autonomous stochastic differential equation

$$
d X_{t}=a\left(X_{s}\right) d s+b\left(X_{s}\right) * d W_{s}, \quad X_{t_{0}}=x_{0},
$$

where $W=\left(\left(W_{t}^{1}, \ldots, W_{t}^{m}\right)\right)_{t \geq 0}$ is an $m$-dimensional Wiener process w.r.t. $\left(\mathcal{F}_{t}\right)_{t \geq 0}$ and the constant $x_{0} \in \mathbb{R}^{d}$ is the initial value. Like in the deterministic setting, each SDE system can be written as an autonomous SDE system with one additional equation representing time. Hence without loss of generality, autonomous stochastic differential equations are treated in the following only. SDE (1) can be written in integral form as

$$
X_{t}=x_{0}+\int_{t_{0}}^{t} a\left(X_{s}\right) d s+\sum_{j=1}^{m} \int_{t_{0}}^{t} b^{j}\left(X_{s}\right) * d W_{s}^{j}
$$

for $t \in I$, where the $j$ th column of the $d \times m$-matrix function $b=\left(b^{i, j}\right)$ is denoted by $b^{j}$ for $j=1, \ldots, m$. The second integral w.r.t. the Wiener process can be interpreted either as an Itô or a Stratonovich integral which is indicated by the asterisk. Thus $* d W$ stands for $d W$ in case of Itô calculus and for $\circ d W$ in case of Stratonovich calculus. We suppose that $a: \mathbb{R}^{d} \rightarrow \mathbb{R}^{d}$ and $b: \mathbb{R}^{d} \rightarrow \mathbb{R}^{d \times m}$ are Borel-measurable and satisfy the conditions of the Existence and Uniqueness Theorem (see, e.g., $[1,6,7,9]$ ).

In order to investigate the Taylor expansion of the expectation of some functional $f: \mathbb{R}^{d} \rightarrow \mathbb{R}$ of the solution $X$ of SDE (2) in the case of Itô calculus, we will make use of the diffusion operator $L^{0}$ and the operators $L^{j}$ for $j=1, \ldots, m$ in case of an $m$-dimensional Wiener process. They are defined as

$$
\begin{aligned}
L^{0} & =\sum_{k=1}^{d} a^{k} \frac{\partial}{\partial x^{k}}+\frac{1}{2} \sum_{k, l=1}^{d} \sum_{j=1}^{m} b^{k, j} b^{l, j} \frac{\partial^{2}}{\partial x^{k} \partial x^{l}} \\
L^{j} & =\sum_{k=1}^{d} b^{k, j} \frac{\partial}{\partial x^{k}} \quad j=1, \ldots, m .
\end{aligned}
$$


Applying Itô's formula recursively to $f\left(X_{t}\right)$ and afterwards to $L^{0} f\left(X_{s}\right)$ yields

$$
\begin{aligned}
f\left(X_{t}\right)= & f\left(X_{t_{0}}\right)+\int_{t_{0}}^{t} L^{0} f\left(X_{s}\right) d s+\sum_{j=1}^{m} \int_{t_{0}}^{t} L^{j} f\left(X_{s}\right) d W_{s}^{j} \\
= & f\left(X_{t_{0}}\right)+\int_{t_{0}}^{t}\left(L^{0} f\left(X_{t_{0}}\right)+\int_{t_{0}}^{s} L^{0} L^{0} f\left(X_{u}\right) d u\right. \\
& \left.+\sum_{j=1}^{m} \int_{t_{0}}^{s} L^{j} L^{0} f\left(X_{u}\right) d W_{u}^{j}\right) d s+\sum_{j=1}^{m} \int_{t_{0}}^{t} L^{j} f\left(X_{s}\right) d W_{s}^{j}
\end{aligned}
$$

This procedure can be continued by applying again Itô's formula to $L^{0} L^{0} f\left(X_{u}\right)$ and so on. In the following, it is always assumed that all of the necessary derivatives and multiple integrals exist. If we consider the case of $d=m=1$ and take the expectation, then we get with $X_{t_{0}}=x_{0} \in \mathbb{R}^{d}$

$$
\begin{aligned}
E^{t_{0}, x_{0}}\left(f\left(X_{t}\right)\right)= & f\left(x_{0}\right)+\left(f^{\prime} a+\frac{1}{2} f^{\prime \prime} b^{2}\right)\left(x_{0}\right) \cdot\left(t-t_{0}\right)+\left(f^{\prime} \cdot\left(a a^{\prime}+\frac{1}{2} b^{2} a^{\prime \prime}\right)\right. \\
& +f^{\prime \prime} \cdot\left(a^{2}+a b b^{\prime}+b^{2} a^{\prime}+\frac{1}{2} b^{2} b^{\prime 2}+\frac{1}{2} b^{3} b^{\prime \prime}\right) \\
& \left.+f^{\prime \prime \prime \prime} \cdot\left(a b^{2}+b^{3} b^{\prime}\right)+f^{\prime \prime \prime \prime} \cdot\left(\frac{1}{4} b^{4}\right)\right)\left(x_{0}\right) \cdot \frac{\left(t-t_{0}\right)^{2}}{2 !}+\ldots
\end{aligned}
$$

since the expectation of a multiple Itô integral with at least one integration w.r.t. a Wiener process vanishes $[6,8]$. The terms on the right hand side, which are called elementary differentials in numerical analysis, get more and more complex for expansions of higher orders. Thus, an approach similar to the rooted tree theory for deterministic ordinary differential equations as described by Butcher [4] is helpful.

\section{Rooted Tree Theory}

First of all a definition of the coloured graphs which will be suitable in the rooted tree theory for stochastic differential equation systems is given. In contrast to the trees introduced by Burrage and Burrage [2,3], the trees defined in the following own an additional kind of node corresponding to the functional and have a particular structure.

Definition 2.1 Let $l$ be a positive integer.

(1) A monotonically labelled S-tree (stochastic tree) $\boldsymbol{t}$ with $l=l(\boldsymbol{t})$ nodes is a pair of maps $\boldsymbol{t}=\left(\boldsymbol{t}^{\prime}, \boldsymbol{t}^{\prime \prime}\right)$ with $\boldsymbol{t}^{\prime \prime}=\left(\boldsymbol{t}_{A}^{\prime \prime}, \boldsymbol{t}_{J}^{\prime \prime}\right)$ and

$$
\begin{aligned}
& t^{\prime}:\{2, \ldots, l\} \longrightarrow\{1, \ldots, l-1\} \\
& \boldsymbol{t}_{A}^{\prime \prime}:\{1, \ldots, l\} \longrightarrow A \\
& \boldsymbol{t}_{J}^{\prime \prime}:\{1, \ldots, l\} \longrightarrow J \cup\{0\}
\end{aligned}
$$


so that $\boldsymbol{t}^{\prime}(i)<i$ for $i=2, \ldots, l ;$ A denotes a finite set and $J=\left\{j_{1}, \ldots, j_{s}\right\}$ is a set of indices.

(2) LTS denotes the set of all monotonically labelled $S$-trees. Here two trees $\boldsymbol{t}=\left(\boldsymbol{t}^{\prime}, \boldsymbol{t}^{\prime \prime}\right)$ and $\boldsymbol{u}=\left(\boldsymbol{u}^{\prime}, \boldsymbol{u}^{\prime \prime}\right)$ just differing by their renamed indices $\boldsymbol{t}_{J}^{\prime \prime}$ and $\boldsymbol{u}_{J}^{\prime \prime}$ are considered to be identical if there exists a bijective map

$$
\pi:\left\{j_{1}, \ldots, j_{s}\right\} \cup\{0\} \longrightarrow\left\{j_{1}, \ldots, j_{s}\right\} \cup\{0\} \quad \text { with } \pi(0)=0
$$

so that $\boldsymbol{t}_{J}^{\prime \prime}(i)=\pi\left(\boldsymbol{u}_{J}^{\prime \prime}(i)\right)$ holds for $i=1, \ldots, l$.

Remark 2.2 Thus LTS consists of monotonically labelled trees whereby two trees are assumed to be identical if we can rename the indices $j_{i}$ of the first one to the indices of the second tree, so that nodes having identical indices receive again identical indices and nodes with different indices receive again different indices.

Every monotonically labelled S-tree $\mathbf{t}$ can be represented as a graph, whose nodes are elements of $\{1, \ldots, l(\mathbf{t})\}$ and whose arcs are the pairs $\left(\mathbf{t}^{\prime}(i), i\right)$ for $i=2, \ldots, l(\mathbf{t})$. Here, $\mathbf{t}^{\prime}$ defines a father son relation between the nodes, i.e. $\mathbf{t}^{\prime}(i)$ is the father of the son $i$. Furthermore the colour $\mathbf{t}^{\prime \prime}(i)=\left(\mathbf{t}_{A}^{\prime \prime}(i), \mathbf{t}_{J}^{\prime \prime}(i)\right)$, which consists of one element of the set $A$ and one element of the set $J \cup\{0\}$, is added to the node $i$ for $i=1, \ldots, l(\mathbf{t})$. The node with label 1 is called root and it is always sketched as the lowest node of the graph. If not stated otherwise, we take $A=\{\gamma, \tau, \sigma\}$ in the following. In this case $\gamma=\otimes$ denotes the root, $\tau=0$ is a deterministic node and $\sigma=0$ is a stochastic node. Further we consider a set $J=\left\{j_{1}, \ldots, j_{s}\right\}$ of indices which are taken as variables so that $j_{i} \in\{1, \ldots, m\}$ for $i=1, \ldots, s$. Usually we assign to the root $\gamma$ and to all deterministic nodes $\tau$ the index 0 , which will be omitted in the following. However, we assign to every stochastic node $\sigma$ an index $j_{i}$, which is associated with the $j_{i}$ th component of the corresponding $m$-dimensional Wiener process of the considered SDE. In case of a one-dimensional Wiener process one can omit the set of indices $J$ since $j_{i}=1$ for all $i=1, \ldots, s$ (see also [11]). As an example Figure 1 presents two elements of $L T S$.
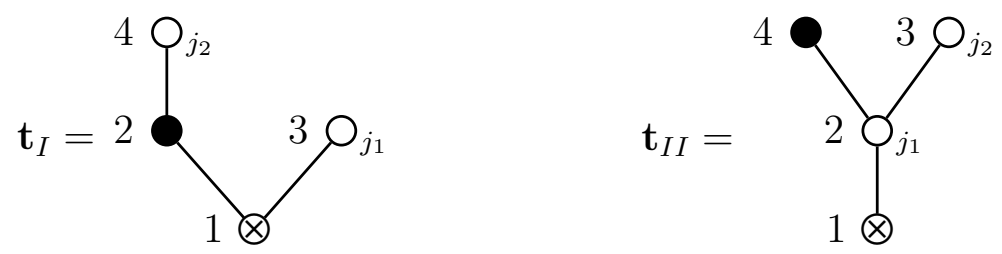

Fig. 1. Two elements of $L T S$ with $j_{1}, j_{2} \in\{1, \ldots, m\}$.

For the labelled S-tree $\mathbf{t}_{I}$ in Figure 1 we have $\mathbf{t}_{I}^{\prime}(2)=\mathbf{t}_{I}^{\prime}(3)=1$ and $\mathbf{t}_{I}^{\prime}(4)=2$. The colour of the nodes is given by $\mathbf{t}_{I}^{\prime \prime}(1)=\gamma, \mathbf{t}_{I}^{\prime \prime}(2)=\tau, \mathbf{t}_{I}^{\prime \prime}(3)=\sigma_{j_{1}}$ and $\mathbf{t}_{I}^{\prime \prime}(4)=\sigma_{j_{2}}$. 
Definition 2.3 Let $\boldsymbol{t}=\left(\boldsymbol{t}^{\prime}, \boldsymbol{t}^{\prime \prime}\right) \in$ LTS w.r.t. the set $A=\{\gamma, \tau, \sigma\}$. Then we denote by $d(\boldsymbol{t})=\sharp\left\{i: \boldsymbol{t}^{\prime \prime}(i)=\tau\right\}$ the number of deterministic nodes and by $s(\boldsymbol{t})=\sharp\left\{i: \boldsymbol{t}^{\prime \prime}(i)=\sigma_{j}, j \in J\right\}$ the number of stochastic nodes of $\boldsymbol{t}$. The order $\rho(\boldsymbol{t})$ of the tree $\boldsymbol{t}$ is defined as $\rho(\boldsymbol{t})=d(\boldsymbol{t})+\frac{1}{2} s(\boldsymbol{t})$ with $\rho(\gamma)=0$.

The order of the trees $\mathbf{t}_{I}$ and $\mathbf{t}_{I I}$ presented in Figure 1 can be calculated as $\rho\left(\mathbf{t}_{I}\right)=\rho\left(\mathbf{t}_{I I}\right)=2$. Every labelled S-tree can be written as a combination of three different brackets defined as follows.

Definition 2.4 If $\boldsymbol{t}_{1}, \ldots, \boldsymbol{t}_{k}$ are coloured trees then we denote by

$$
\left(\boldsymbol{t}_{1}, \ldots, \boldsymbol{t}_{k}\right), \quad\left[\boldsymbol{t}_{1}, \ldots, \boldsymbol{t}_{k}\right] \quad \text { and } \quad\left\{\boldsymbol{t}_{1}, \ldots, \boldsymbol{t}_{k}\right\}_{j}
$$

the tree in which $\boldsymbol{t}_{1}, \ldots, \boldsymbol{t}_{k}$ are each joined by a single branch to $\otimes$, $\bullet$ and $\mathrm{O}_{j}$, respectively (see Figure 2).

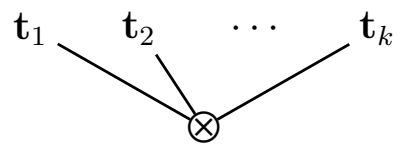

$\left(\mathbf{t}_{1}, \ldots, \mathbf{t}_{k}\right)$

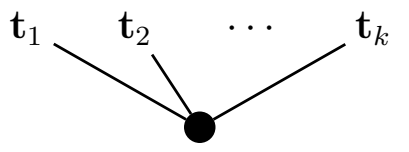

$\left[\mathbf{t}_{1}, \ldots, \mathbf{t}_{k}\right]$

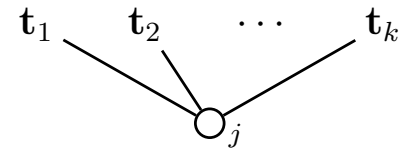

$\left\{\mathbf{t}_{1}, \ldots, \mathbf{t}_{k}\right\}_{j}$

Fig. 2. Writing a coloured S-tree with brackets.

Therefore proceeding recursively, for the two examples $\mathbf{t}_{I}$ and $\mathbf{t}_{I I}$ in Figure 1 we obtain $\mathbf{t}_{I}=\left(\left[\mathrm{O}_{j_{2}}\right], \bigcirc_{j_{1}}\right)=\left(\left[\sigma_{j_{2}}\right], \sigma_{j_{1}}\right)$ and $\mathbf{t}_{I I}=\left(\left\{\boldsymbol{\bullet}, \bigcirc_{j_{2}}\right\}_{j_{1}}\right)=\left(\left\{\tau, \sigma_{j_{2}}\right\}_{j_{1}}\right)$.

In the following, we will concentrate our considerations to one representative tree of each equivalence class defined as follows:

Definition 2.5 Let $\boldsymbol{t}=\left(\boldsymbol{t}^{\prime}, \boldsymbol{t}^{\prime \prime}\right)$ and $\boldsymbol{u}=\left(\boldsymbol{u}^{\prime}, \boldsymbol{u}^{\prime \prime}\right)$ be elements of LTS. Then the trees $\boldsymbol{t}$ and $\boldsymbol{u}$ are equivalent, i.e. $\boldsymbol{t} \sim \boldsymbol{u}$, if the following hold:

(i) $l(\boldsymbol{t})=l(\boldsymbol{u})$

(ii) There exist two bijective maps

$$
\begin{aligned}
& \psi:\{1, \ldots, l(\boldsymbol{t})\} \rightarrow\{1, \ldots, l(\boldsymbol{t})\} \quad \text { with } \quad \psi(1)=1, \\
& \pi:\left\{j_{1}, \ldots, j_{s}\right\} \cup\{0\} \rightarrow\left\{j_{1}, \ldots, j_{s}\right\} \cup\{0\} \quad \text { with } \pi(0)=0
\end{aligned}
$$

so that the following diagram commutes

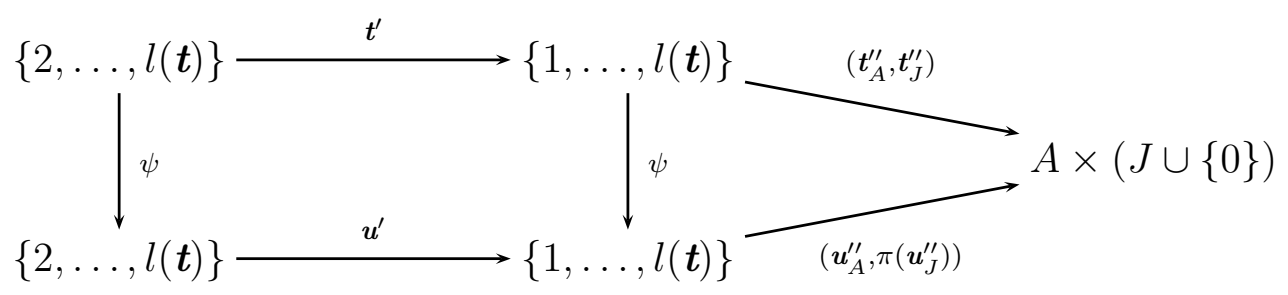


Thus, a monotonically labelled S-tree $\mathbf{u}$ is equivalent to $\mathbf{t}$, if each label $k$ is replaced by $\psi(k)$ and if the variable indices $j_{i}$ are replaced by $\pi\left(j_{i}\right)$. Now it is straight forward to consider equivalence classes.

Definition 2.6 The set of all equivalence classes under the relation of Definition 2.5 is denoted by $T S=L T S / \sim$. The elements of TS are called (rooted) S-trees. We denote by $\alpha(\boldsymbol{t})$ the cardinality of $\boldsymbol{t}$, i.e. the number of possibilities of monotonically labelling the nodes of $\boldsymbol{t}$ with numbers $1, \ldots, l(\boldsymbol{t})$.

Thus, all trees in Figure 3 belong to the same equivalence class as $\mathbf{t}_{I}$ in the example above, since the indices $j_{1}$ and $j_{2}$ are just renamed either by $j_{2}$ and $j_{1}$ or $j_{8}$ and $j_{3}$, respectively. Finally the graphs differ only in the labelling of their number indices.
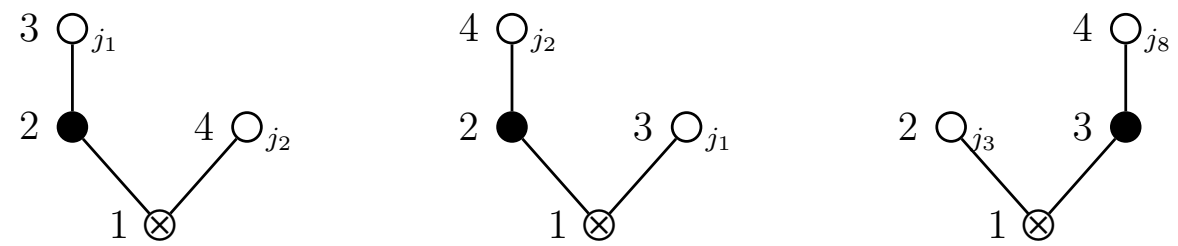

Fig. 3. Trees of the same equivalence class.

For every rooted tree $\mathbf{t} \in L T S$, there exists a corresponding elementary differential which is a direct generalization of the differential in the deterministic case (see, e.g., [4]). For $j \in\{1, \ldots, m\}$, the elementary differential is defined recursively by

$$
F(\gamma)(x)=f(x), \quad F(\tau)(x)=a(x), \quad F\left(\sigma_{j}\right)(x)=b^{j}(x),
$$

for single nodes and by

$$
F(\mathbf{t})(x)= \begin{cases}f^{(k)}(x) \cdot\left(F\left(\mathbf{t}_{1}\right)(x), \ldots, F\left(\mathbf{t}_{k}\right)(x)\right) & \text { for } \mathbf{t}=\left(\mathbf{t}_{1}, \ldots, \mathbf{t}_{k}\right) \\ a^{(k)}(x) \cdot\left(F\left(\mathbf{t}_{1}\right)(x), \ldots, F\left(\mathbf{t}_{k}\right)(x)\right) & \text { for } \mathbf{t}=\left[\mathbf{t}_{1}, \ldots, \mathbf{t}_{k}\right] \\ b^{(k)}(x) \cdot\left(F\left(\mathbf{t}_{1}\right)(x), \ldots, F\left(\mathbf{t}_{k}\right)(x)\right) & \text { for } \mathbf{t}=\left\{\mathbf{t}_{1}, \ldots, \mathbf{t}_{k}\right\}_{j}\end{cases}
$$

for a tree $\mathbf{t}$ with more than one node. Here $f^{(k)}, a^{(k)}$ and $b^{j^{(k)}}$ define a symmetric $k$-linear differential operator, and one can choose the sequence of labelled S-trees $\mathbf{t}_{1}, \ldots, \mathbf{t}_{k}$ in an arbitrary order. For example, the $I$ th component of $a^{(k)} \cdot\left(F\left(\mathbf{t}_{1}\right), \ldots, F\left(\mathbf{t}_{k}\right)\right)$ can be written as

$$
\left(a^{(k)} \cdot\left(F\left(\mathbf{t}_{1}\right), \ldots, F\left(\mathbf{t}_{k}\right)\right)\right)^{I}=\sum_{J_{1}, \ldots, J_{k}=1}^{d} \frac{\partial^{k} a^{I}}{\partial x^{J_{1}} \ldots \partial x^{J_{k}}}\left(F^{J_{1}}\left(\mathbf{t}_{1}\right), \ldots, F^{J_{k}}\left(\mathbf{t}_{k}\right)\right)
$$

where the components of vectors are denoted by superscript indices, which are chosen as capitals. As a result of this we get for $\mathbf{t}_{I}$ and $\mathbf{t}_{I I}$ the elementary 
differentials

$$
\begin{aligned}
& F\left(\mathbf{t}_{I}\right)=f^{\prime \prime}\left(a^{\prime}\left(b^{j_{2}}\right), b^{j_{1}}\right)=\sum_{J_{1}, J_{2}=1}^{d} \frac{\partial^{2} f}{\partial x^{J_{1}} \partial x^{J_{2}}}\left(\sum_{K_{1}=1}^{d} \frac{\partial a^{J_{1}}}{\partial x^{K_{1}}} b^{K_{1}, j_{2}} \cdot b^{J_{2}, j_{1}}\right) \\
& F\left(\mathbf{t}_{I I}\right)=f^{\prime}\left(b^{j_{1} \prime}\left(a, b^{j_{2}}\right)\right)=\sum_{J_{1}=1}^{d} \frac{\partial f}{\partial x^{J_{1}}}\left(\sum_{K_{1}, K_{2}=1}^{d} \frac{\partial^{2} b^{J_{1}, j_{1}}}{\partial x^{K_{1}} \partial x^{K_{2}}} a^{K_{1}} \cdot b^{K_{2}, j_{2}}\right)
\end{aligned}
$$

It has to be pointed out that the elementary differentials for the trees presented in Figure 3 coincide with $F\left(\mathbf{t}_{I}\right)$ if the variable indices $j_{i}$ are simply renamed by a suitable bijective mapping $\pi$.

There exists a close relation between differentiation of an elementary differential and the growth of the corresponding labelled tree, that is adding further nodes to the tree.

Lemma 2.7 Let $\boldsymbol{t}=\left(\boldsymbol{t}^{\prime}, \boldsymbol{t}^{\prime \prime}\right) \in L T S$ and let $\lambda=l(\boldsymbol{t})$ denote the number of nodes of $\boldsymbol{t}$. Then for $g \equiv a$ or $g \equiv b^{j}$ and $x \in \mathbb{R}^{d}$

$$
\sum_{k=1}^{d} g^{k}(x) \frac{\partial}{\partial x^{k}} F(\boldsymbol{t})(x)=\sum_{\boldsymbol{u} \in H_{1}(\boldsymbol{t})} F(\boldsymbol{u})(x)
$$

holds, where $H_{1}(\boldsymbol{t})$ is the set of trees $\boldsymbol{u}=\left(\boldsymbol{u}^{\prime}, \boldsymbol{u}^{\prime \prime}\right) \in$ LTS with $l(\boldsymbol{u})=\lambda+1$ nodes, $\left.\boldsymbol{u}^{\prime}\right|_{\{2, \ldots, \lambda\}}=\boldsymbol{t}^{\prime},\left.\boldsymbol{u}^{\prime \prime}\right|_{\{1, \ldots, \lambda\}}=\boldsymbol{t}^{\prime \prime}$ and either $\boldsymbol{u}^{\prime \prime}(\lambda+1)=\tau$ in case of $g \equiv a$ or $\boldsymbol{u}^{\prime \prime}(\lambda+1)=\sigma_{j}$ in case of $g \equiv b^{j}$.

Proof. $F(\mathbf{t})(x)$ is by definition a product of some derivatives of the functions $f, a$ and $b^{j}$. So if we apply the operator $\sum_{k=1}^{d} g^{k} \frac{\partial}{\partial x^{k}}$ to $F(\mathbf{t})$, we have to use the product rule for differentiation. Thus we get a sum with exactly $\lambda=l(\mathbf{t})$ summands. Now, we only have to observe that each of these summands is equal to the elementary differential of the tree $\mathbf{t}$ with one additional arc $(\lambda+1, i)$ and a new node $\lambda+1$ of type $\tau$ in case of $g \equiv a$ or of type $\sigma_{j}$ in case of $g \equiv b^{j}$ for the corresponding $i \in\{1, \ldots, \lambda\}$, respectively.

The growth of an $S$-tree which corresponds to the twice repeated differentiation of the elementary differential, is stated by the following lemma.

Lemma 2.8 Let $\boldsymbol{t}=\left(\boldsymbol{t}^{\prime}, \boldsymbol{t}^{\prime \prime}\right) \in L T S$ and let $\lambda=l(\boldsymbol{t})$ denote the number of nodes of $\boldsymbol{t}$. Then for $x \in \mathbb{R}^{d}$

$$
\sum_{k, l=1}^{d} b^{k, j}(x) b^{l, j}(x) \frac{\partial^{2}}{\partial x^{k} \partial x^{l}} F(\boldsymbol{t})(x)=\sum_{\boldsymbol{u} \in H_{2}(\boldsymbol{t})} F(\boldsymbol{u})(x)
$$

holds, where $H_{2}(\boldsymbol{t})$ is the set of trees $\boldsymbol{u}=\left(\boldsymbol{u}^{\prime}, \boldsymbol{u}^{\prime \prime}\right) \in$ LTS with $l(\boldsymbol{u})=\lambda+2$ nodes, $\left.\boldsymbol{u}^{\prime}\right|_{\{2, \ldots, \lambda\}}=\boldsymbol{t}^{\prime},\left.\boldsymbol{u}^{\prime \prime}\right|_{\{1, \ldots, \lambda\}}=\boldsymbol{t}^{\prime \prime}, \boldsymbol{u}^{\prime \prime}(\lambda+1)=\boldsymbol{u}^{\prime \prime}(\lambda+2)=\sigma_{j}$ and $\boldsymbol{u}^{\prime}(\lambda+2) \neq$ $\lambda+1$. 
Proof. Again, $F(\mathbf{t})(x)$ is by definition a product of some derivatives of the functions $f, a$ and $b^{j}$. So if we apply the operator $\sum_{k, l=1}^{d} b^{k, j} b^{l, j} \frac{\partial^{2}}{\partial x^{k} \partial x^{l}}$ to $F(\mathbf{t})$, we have to use the product rule for differentiation. Thus we obtain a sum with exactly $\lambda^{2}=l(\mathbf{t})^{2}$ summands. Now, we only have to observe that each of these summands is equal to the elementary differential of the tree $\mathbf{t}$ with two additional arcs $(\lambda+1, r)$ and $(\lambda+2, s)$ and two new nodes $\lambda+1$ and $\lambda+2$, both of type $\sigma_{j}$, for the corresponding $r, s \in\{1, \ldots, \lambda\}$, respectively. It has to be pointed out, that $r=s$ or $r \neq s$ is possible.

In the following, let $C_{P}^{l}\left(\mathbb{R}^{d}, \mathbb{R}\right)$ denote the space of $l$ times continuously differentiable functions $g \in C^{l}\left(\mathbb{R}^{d}, \mathbb{R}\right)$ for which all partial derivatives up to order $l$ have polynomial growth. That is, for which there exist constants $K>0$ and $r \in \mathbb{N}$ depending on $g$, such that

$$
\left|\partial_{x}^{i} g(x)\right| \leq K\left(1+\|x\|^{2 r}\right)
$$

holds for all $x \in \mathbb{R}^{d}$ and any partial derivative $\partial_{x}^{i} g$ of order $i \leq l$.

\section{Truncated Taylor expansions for Itô SDE Systems}

For the expansion of the expectation of some functional of the diffusion process $\left(X_{t}\right)_{t \in I}$ which is solution of the $d$-dimensional Itô SDE

$$
X_{t}=x_{0}+\int_{t_{0}}^{t} a\left(X_{s}\right) d s+\sum_{j=1}^{m} \int_{t_{0}}^{t} b^{j}\left(X_{s}\right) d W_{s}^{j}
$$

with constant initial value $x_{0} \in \mathbb{R}^{d}$ we have to introduce the subset $\operatorname{LTS}(I)$ of $L T S$.

Definition 3.1 Let LTS $(I)$ denote the set of trees $\boldsymbol{t}=\left(\boldsymbol{t}^{\prime}, \boldsymbol{t}^{\prime \prime}\right) \in$ LTS having as root $\gamma=\otimes$ and which can be constructed by a finite number of steps of the form

a) adding a deterministic node $\tau=\mathbf{0}$, or

b) adding two stochastic nodes $\sigma_{j}=\bigcirc_{j}$, where both have the same variable index $j \in J$ and neither of them is father of the other.

The nodes have to be labelled in the same order as they have been added by the construction of the tree. Further $T S(I)=L T S(I) / \sim$ denotes the equivalence class under the relation of Definition 2.5 restricted to LTS $(I)$ and $\alpha_{I}(\boldsymbol{t})$ denotes the cardinality of $\boldsymbol{t}$ in $L T S(I)$.

Since the number of stochastic nodes is always even, the order $\rho(\mathbf{t})$ has to be an integer and $\mathbf{t}$ owns the variable indices $j_{1}, \ldots, j_{s(\mathbf{t}) / 2}$. See Figure 4 for some 
examples of $T S(I)$ up to order two.

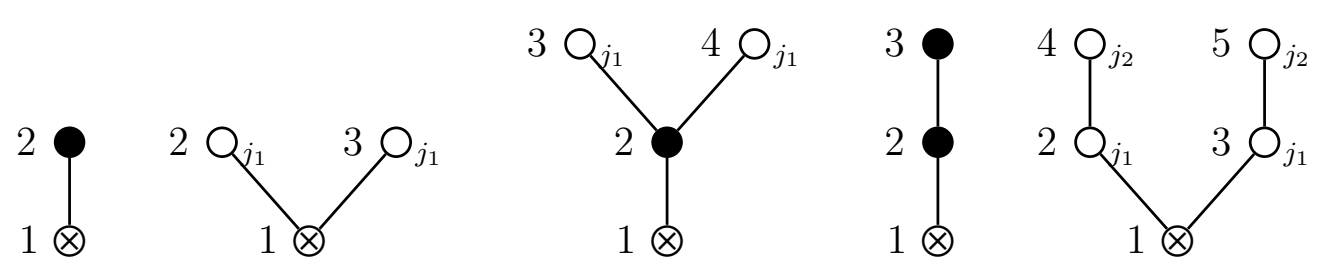

Fig. 4. Some trees which belong to $\operatorname{LTS}(I)$.

Now the first main result providing a compact way of representing the expectation of some functional of the solution of an Itô stochastic differential equation can be stated.

Theorem 3.2 Let $\left(X_{t}\right)_{t \in I}$ be the solution of the Itô stochastic differential equation system (11) with initial value $X_{t_{0}}=x_{0} \in \mathbb{R}^{d}$. Then for $n \in \mathbb{N}_{0}$ and $f, a^{i}, b^{i, j} \in C_{P}^{2(n+1)}\left(\mathbb{R}^{d}, \mathbb{R}\right)$ for $i=1, \ldots, d$ and $j=1, \ldots, m$ we get the following:

(1) For every $t \in\left[t_{0}, T\right]$ the following truncated expansion holds:

$$
\begin{aligned}
E^{t_{0}, x_{0}}\left(f\left(X_{t}\right)\right) & =\sum_{\substack{\boldsymbol{t} \in L T S(I) \\
\rho(\boldsymbol{t}) \leq n}} \sum_{j_{1}, \ldots, j_{s(t) / 2}=1}^{m} \frac{F(\boldsymbol{t})\left(x_{0}\right)}{2^{s(\boldsymbol{t}) / 2} \rho(\boldsymbol{t}) !}\left(t-t_{0}\right)^{\rho(\boldsymbol{t})}+\mathcal{R}_{n}\left(t, t_{0}\right) \\
& =\sum_{\substack{\boldsymbol{t} \in T S(I) \\
\rho(\boldsymbol{t}) \leq n}} \sum_{j_{1}, \ldots, j_{s(t) / 2}=1}^{m} \frac{\alpha_{I}(\boldsymbol{t}) F(\boldsymbol{t})\left(x_{0}\right)}{2^{s(\boldsymbol{t}) / 2} \rho(\boldsymbol{t}) !}\left(t-t_{0}\right)^{\rho(\boldsymbol{t})}+\mathcal{R}_{n}\left(t, t_{0}\right)
\end{aligned}
$$

(2) There exists a random variable $\xi: \Omega \rightarrow] t_{0}, t[$ so that holds:

$$
\begin{aligned}
\mathcal{R}_{n}\left(t, t_{0}\right) & =\sum_{\substack{t \in L T S(I) \\
\rho(\boldsymbol{t})=n+1}} \sum_{j_{1}, \ldots, j_{s(t) / 2}=1}^{m} \frac{E^{t_{0}, x_{0}}\left(F(\boldsymbol{t})\left(X_{\xi}\right)\right)}{2^{s(\boldsymbol{t}) / 2} \rho(\boldsymbol{t}) !}\left(t-t_{0}\right)^{\rho(\boldsymbol{t})} \\
& =\sum_{\substack{\boldsymbol{t} \in T S(I) \\
\rho(\boldsymbol{t})=n+1}} \sum_{j_{1}, \ldots, j_{s(t) / 2}=1}^{m} \frac{\alpha_{I}(\boldsymbol{t}) E^{t_{0}, x_{0}}\left(F(\boldsymbol{t})\left(X_{\xi}\right)\right)}{2^{s(\boldsymbol{t}) / 2} \rho(\boldsymbol{t}) !}\left(t-t_{0}\right)^{\rho(\boldsymbol{t})}
\end{aligned}
$$

Proof. Consider the diffusion operator $11 L^{0}$ and the operator $L^{j}$ defined by (3). Then, by reapplication of Itô's formula as in (4) we get

$$
E^{t_{0}, x_{0}}\left(f\left(X_{t}\right)\right)=\sum_{i=0}^{n} \frac{\left(L^{0}\right)^{i} f\left(x_{0}\right)}{i !}\left(t-t_{0}\right)^{i}+\mathcal{R}_{n}^{*}\left(t, t_{0}\right)
$$

$\overline{1 L^{0} \text { is }}$ also called the generator of the solution $X$ (see, e.g., $\left.[5,9,12]\right)$. 
with

$$
\mathcal{R}_{n}^{*}\left(t, t_{0}\right)=E^{t_{0}, x_{0}}\left(\int_{t_{0}}^{t} \int_{t_{0}}^{s_{n}} \ldots \int_{t_{0}}^{s_{1}}\left(L^{0}\right)^{n+1} f\left(X_{s}\right) d s d s_{1} \ldots d s_{n}\right)
$$

Here, it should be remarked that the first moment of a multiple Itô integral vanishes if it has at least one integration w.r.t. a Wiener process (see [8]). Thus, in order to prove (12) we have to show the following equation

$$
\sum_{i=0}^{n} \frac{\left(L^{0}\right)^{i} f\left(x_{0}\right)}{i !}\left(t-t_{0}\right)^{i}=\sum_{\substack{\mathbf{t} \in L T S(I) \\ \rho(\mathbf{t}) \leq n}} \sum_{j_{1}, \ldots, j_{s(\mathbf{t}) / 2}=1}^{m} \frac{F(\mathbf{t})\left(x_{0}\right)}{2^{s(\mathbf{t}) / 2} \rho(\mathbf{t}) !}\left(t-t_{0}\right)^{\rho(\mathbf{t})}
$$

Therefore, for every $n \in \mathbb{N}_{0}$ we finally have to prove

$$
\left(L^{0}\right)^{n} f\left(x_{0}\right)=\sum_{\substack{\mathbf{t} \in L T S(I) \\ \rho(\mathbf{t})=n}} \sum_{j_{1}, \ldots, j_{s}(\mathbf{t}) / 2}^{m} \frac{F(\mathbf{t})\left(x_{0}\right)}{2^{s(\mathbf{t}) / 2}} .
$$

We proceed by induction. The case $n=0$ is trivial: There is only one element $\gamma=\otimes$ in $\operatorname{LTS}(I)$ with $\rho(\gamma)=0$ and we get with $\left(L^{0}\right)^{0} \equiv$ Id that $\left(L^{0}\right)^{0} f\left(x_{0}\right)=f\left(x_{0}\right)=F(\gamma)\left(x_{0}\right)$. The step $n=1$ is performed as well for better understanding. In this case two different trees $\mathbf{t}_{1.1}=(\tau)$ and $\mathbf{t}_{1.2}=\left(\sigma_{j_{1}}, \sigma_{j_{1}}\right)$ in $\operatorname{LTS}(I)$, both of order 1 , have to be considered. For these two trees we have

$$
\begin{aligned}
L^{0}\left(F(\gamma)\left(x_{0}\right)\right) & =\left(\sum_{k=1}^{d} a^{k} \frac{\partial f}{\partial x^{k}}+\frac{1}{2} \sum_{k, l=1}^{d} \sum_{j=1}^{m} b^{k, j} b^{l, j} \frac{\partial^{2} f}{\partial x^{k} \partial x^{l}}\right)\left(x_{0}\right) \\
& =F\left(\mathbf{t}_{1.1}\right)\left(x_{0}\right)+\frac{1}{2} \sum_{j_{1}=1}^{m} F\left(\mathbf{t}_{1.2}\right)\left(x_{0}\right) \\
& =\sum_{\substack{\mathbf{t} \in L T S(I) \\
\rho(\mathbf{t})=1}} \sum_{j_{1}, \ldots, j_{s(\mathbf{t}) / 2}=1}^{m} \frac{F(\mathbf{t})\left(x_{0}\right)}{2^{s(\mathbf{t}) / 2}} .
\end{aligned}
$$

Under the assumption that equation (17) holds for $n \in \mathbb{N}_{0}$ we now proceed to give a prove for the case $n+1$. Therefore, we get

$$
\begin{aligned}
\left(L^{0}\right)^{n+1} f\left(x_{0}\right)= & \left(L^{0}\right)\left(L^{0}\right)^{n} f\left(x_{0}\right)=\left(L^{0}\right) \sum_{\substack{\mathbf{t} \in L T S(I) \\
\rho(\mathbf{t})=n}} \sum_{j_{1}, \ldots, j_{s(\mathbf{t}) / 2}=1}^{m} \frac{F(\mathbf{t})\left(x_{0}\right)}{2^{s(\mathbf{t}) / 2}} \\
= & \sum_{\substack{\mathbf{t} \in L T S(I) \\
\rho(\mathbf{t})=n}} \sum_{j_{1}, \ldots, j_{s(\mathbf{t}) / 2}=1}^{m} \frac{1}{2^{s(\mathbf{t}) / 2}}\left(\sum_{k=1}^{d} a^{k} \frac{\partial}{\partial x^{k}} F(\mathbf{t})\right. \\
& \left.+\frac{1}{2} \sum_{k, l=1}^{d} \sum_{j=1}^{m} b^{k, j} b^{l, j} \frac{\partial^{2}}{\partial x^{k} \partial x^{l}} F(\mathbf{t})\right)\left(x_{0}\right) .
\end{aligned}
$$


Next consider the first part of the operator $L^{0}$ and apply Lemma 2.7. Then for $\mathbf{u} \in \operatorname{LTS}(I)$ with $\rho(\mathbf{u})=n$ and $l(\mathbf{u})=\lambda$ we get

$$
\sum_{k=1}^{d} a^{k}\left(x_{0}\right) \frac{\partial}{\partial x^{k}} \frac{F(\mathbf{u})\left(x_{0}\right)}{2^{s(\mathbf{u}) / 2}}=\sum_{\mathbf{t} \in H_{1}(\mathbf{u})} \frac{F(\mathbf{t})\left(x_{0}\right)}{2^{s(\mathbf{t}) / 2}}
$$

where $H_{1}(\mathbf{u})$ is the set of all trees $\mathbf{t} \in \operatorname{LT} S(I)$ with $\rho(\mathbf{t})=n+1,\left.\mathbf{t}^{\prime}\right|_{\{2, \ldots, \lambda\}}=\mathbf{u}^{\prime}$, $\left.\mathbf{t}^{\prime \prime}\right|_{\{1, \ldots, \lambda\}}=\mathbf{u}^{\prime \prime}$ and $\mathbf{t}^{\prime \prime}(\lambda+1)=\tau$. Clearly $s(\mathbf{u})=s(\mathbf{t})$ holds for all $\mathbf{t} \in H_{1}(\mathbf{u})$.

Now we proceed by considering the second part of the operator $L^{0}$. Having Lemma 2.8 in mind, for $\mathbf{u} \in \operatorname{LTS}(I)$ with $\rho(\mathbf{u})=n$ and $l(\mathbf{u})=\lambda$ we calculate

$$
\begin{aligned}
& \frac{1}{2} \sum_{k, l=1}^{d} \sum_{j=1}^{m} b^{k, j}\left(x_{0}\right) b^{l, j}\left(x_{0}\right) \frac{\partial^{2}}{\partial x^{k} \partial x^{l}} \sum_{j_{1}, \ldots, j_{s(\mathbf{u}) / 2}=1}^{m} \frac{F(\mathbf{u})\left(x_{0}\right)}{2^{s(\mathbf{u}) / 2}} \\
& =\sum_{\mathbf{t} \in H_{2}(\mathbf{u})} \sum_{j_{1}, \ldots, j_{s(\mathbf{t}) / 2}=1}^{m} \frac{F(\mathbf{t})\left(x_{0}\right)}{2^{s(\mathbf{t}) / 2}}
\end{aligned}
$$

where $H_{2}(\mathbf{u})$ is the set of all trees $\mathbf{t}=\left(\mathbf{t}^{\prime}, \mathbf{t}^{\prime \prime}\right) \in \operatorname{LTS}(I)$ with $l(\mathbf{t})=\lambda+2$ nodes, of order $\rho(\mathbf{t})=n+1$, with $\left.\mathbf{t}^{\prime}\right|_{\{2, \ldots, \lambda\}}=\mathbf{u}^{\prime},\left.\mathbf{t}^{\prime \prime}\right|_{\{1, \ldots, \lambda\}}=\mathbf{u}^{\prime \prime}$ and $\mathbf{t}^{\prime \prime}(\lambda+1)=$ $\mathbf{t}^{\prime \prime}(\lambda+2)=\sigma_{j}=\sigma_{j_{s(\mathbf{u}) / 2+1}}=\sigma_{j_{s(\mathbf{t}) / 2}}$ since $s(\mathbf{t})=s(\mathbf{u})+2$ for all $\mathbf{t} \in H_{2}(\mathbf{u})$.

Taking together the results (19) and (20), the following equation

$$
\begin{aligned}
\left(L^{0}\right) \sum_{j_{1}, \ldots, j_{s(\mathbf{u}) / 2}=1}^{m} \frac{F(\mathbf{u})\left(x_{0}\right)}{2^{s(\mathbf{u}) / 2}=} & \sum_{\mathbf{t} \in H_{1}(\mathbf{u})} \sum_{j_{1}, \ldots, j_{s(\mathbf{t}) / 2}=1}^{m} \frac{F(\mathbf{t})\left(x_{0}\right)}{2^{s(\mathbf{t}) / 2}} \\
& +\sum_{\mathbf{t} \in H_{2}(\mathbf{u})} \sum_{j_{1}, \ldots, j_{s(\mathbf{t}) / 2}=1}^{m} \frac{F(\mathbf{t})\left(x_{0}\right)}{2^{s(\mathbf{t}) / 2}} \\
= & \sum_{\mathbf{t} \in H_{1}(\mathbf{u}) \cup H_{2}(\mathbf{u})} \sum_{j_{1}, \ldots, j_{s(\mathbf{t}) / 2}=1}^{m} \frac{F(\mathbf{t})\left(x_{0}\right)}{2^{s(\mathbf{t}) / 2}}
\end{aligned}
$$

holds for every $\mathbf{u} \in \operatorname{LTS}(I)$ with $\rho(\mathbf{u})=n$. Now it is easily seen that

$$
\bigcup_{\substack{\mathbf{u} \in L T S(I) \\ \rho(\mathbf{u})=n}}\left(H_{1}(\mathbf{u}) \cup H_{2}(\mathbf{u})\right)=\{\mathbf{t} \in \operatorname{LTS}(I): \rho(\mathbf{t})=n+1\} .
$$

By applying (21) and (22) to equation (18) we arrive at

$$
\left(L^{0}\right) \sum_{\substack{\mathbf{t} \in L T S(I) \\ \rho(\mathbf{t})=n}} \sum_{j_{1}, \ldots, j_{s(\mathbf{t}) / 2}=1}^{m} \frac{F(\mathbf{t})\left(x_{0}\right)}{2^{s(\mathbf{t}) / 2}}=\sum_{\substack{\mathbf{t} \in L T S(I) \\ \rho(\mathbf{t})=n+1}} \sum_{j_{1}, \ldots, j_{s(\mathbf{t}) / 2}=1}^{m} \frac{F(\mathbf{t})\left(x_{0}\right)}{2^{s(\mathbf{t}) / 2}}
$$

which completes the proof of the first part of Theorem 3.2.

Finally, we have to prove that $\mathcal{R}_{n}\left(t, t_{0}\right)=\mathcal{R}_{n}^{*}\left(t, t_{0}\right)$. Due to the Existence 
and Uniqueness Theorem, there exists $N \subset \Omega$ with $P(N)=0$ such that for all $\omega \in \Omega \backslash N$ the solution $X_{t}(\omega)$ of the Ito SDE (2) is a continuous function of $t$. Now $f, a^{i}, b^{i, j} \in C_{P}^{2(n+1)}\left(\mathbb{R}^{d}, \mathbb{R}\right)$ for $i=1, \ldots, d$ and $j=1, \ldots, m$ implies that $\left(L^{0}\right)^{n+1} f \in C_{P}\left(\mathbb{R}^{d}, \mathbb{R}\right)$. Then for every $\omega \in \Omega \backslash N$ there exists $\left.\xi(\omega) \in\right] t_{0}, t[$ so that

$$
\int_{t_{0}}^{t} \int_{t_{0}}^{s_{n}} \ldots \int_{t_{0}}^{s_{1}}\left(L^{0}\right)^{n+1} f\left(X_{s}(\omega)\right) d s d s_{1} \ldots d s_{n}=\frac{\left(L^{0}\right)^{n+1} f\left(X_{\xi}(\omega)\right)}{(n+1) !}\left(t-t_{0}\right)^{n+1}
$$

holds. Applying (17) and taking the expectation completes the proof.

A spreadsheet containing all trees $\mathbf{t}$ of the set $T S(I)$ up to order 2.0 together with the corresponding graph can be found in Table 1.

\section{Truncated Taylor expansions for Stratonovich SDE Systems}

An expansion of the expectation of some functional applied to the solution of a Stratonovich SDE system can be stated in a similar way as introduced for Itô SDE systems. Therefore we consider the following SDE w.r.t. Stratonovich calculus

$$
X_{t}=x_{0}+\int_{t_{0}}^{t} \underline{a}\left(X_{s}\right) d s+\sum_{j=1}^{m} \int_{t_{0}}^{t} b^{j}\left(X_{s}\right) \circ d W_{s} .
$$

The solution $\left(X_{t}\right)_{t \in I}$ of the Stratonovich SDE is also solution of a corresponding Itô SDE as in (11) and therefore also a diffusion process, however with modified drift

$$
\tilde{a}^{i}(x)=\underline{a}^{i}(x)+\frac{1}{2} \sum_{k=1}^{d} \sum_{l=1}^{m} b^{k, l}(x) \frac{\partial b^{i, l}}{\partial x^{k}}(x)
$$

for $i=1, \ldots, d$. Again we introduce a special subset $L T S(S)$ of $L T S$.

Definition 4.1 Let LTS $(S)$ denote the set of trees $\boldsymbol{t}=\left(\boldsymbol{t}^{\prime}, \boldsymbol{t}^{\prime \prime}\right) \in L T S$ having as root $\gamma=\otimes$ and which can be constructed by a finite number of steps of the form

a) adding a deterministic node $\tau=\mathbf{0}$, or

b) adding two stochastic nodes $\sigma_{j}=\bigcirc_{j}$, where both nodes have the same variable index $j \in J$.

The nodes have to be labelled in the same order as they have been added by the construction of the tree. Further $T S(S)=L T S(S) / \sim$ denotes the equivalence class under the relation of Definition 2.5 restricted to LTS $S)$ and $\alpha_{S}(\boldsymbol{t})$ denotes the cardinality of $\boldsymbol{t}$ in $\operatorname{LTS}(S)$. 
The construction of the trees $\mathbf{t} \in L T S(S)$ forces the number of stochastic nodes to be even, the order $\rho(\mathbf{t})$ has to be an integer and $\mathbf{t}$ owns the variable indices $j_{1}, \ldots, j_{s(\mathbf{t}) / 2}$. Figure 5 presents some examples up to order two of the set $T S(S)$. As the construction of the trees in $L T S(I)$ is more restrictive than the ones in $\operatorname{LTS}(S)$, we have $\operatorname{LTS}(I) \subset L T S(S)$. As an example, the first and the last tree of Figure 5 don't belong to $T S(I)$.
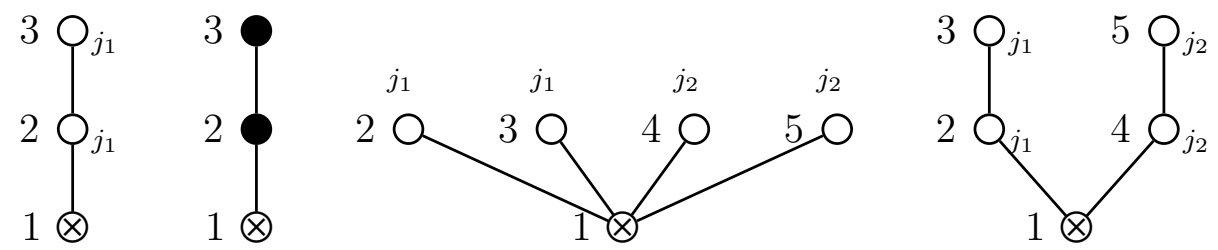

Fig. 5. Some trees which belong to $T S(S)$.

The following result can be proved in nearly the same way as Theorem 3.2.

Theorem 4.2 Let $\left(X_{t}\right)_{t \in I}$ be the solution of the Stratonovich stochastic differential equation system (24) with initial value $X_{t_{0}}=x_{0} \in \mathbb{R}^{d}$. Then for $n \in \mathbb{N}_{0}$ and $f, \tilde{a}^{i}, b^{i, j} \in C_{P}^{2(n+1)}\left(\mathbb{R}^{d}, \mathbb{R}\right)$ for $i=1, \ldots, d$ and $j=1, \ldots, m$ we get the following:

(1) For every $t \in\left[t_{0}, T\right]$ the following truncated expansion holds:

$$
\begin{aligned}
E^{t_{0}, x_{0}}\left(f\left(X_{t}\right)\right) & =\sum_{\substack{\boldsymbol{t} \in L T S(S) \\
\rho(\boldsymbol{t}) \leq n}} \sum_{j_{1}, \ldots, j_{s(t) / 2}=1}^{m} \frac{F(\boldsymbol{t})\left(x_{0}\right)}{2^{s(\boldsymbol{t}) / 2} \rho(\boldsymbol{t}) !}\left(t-t_{0}\right)^{\rho(\boldsymbol{t})}+\underline{\mathcal{R}}_{n}\left(t, t_{0}\right) \\
& =\sum_{\substack{\boldsymbol{t} \in T S(S) \\
\rho(\boldsymbol{t}) \leq n}} \sum_{j_{1}, \ldots, j_{s(t) / 2}=1}^{m} \frac{\alpha_{S}(\boldsymbol{t}) F(\boldsymbol{t})\left(x_{0}\right)}{2^{s(\boldsymbol{t}) / 2} \rho(\boldsymbol{t}) !}\left(t-t_{0}\right)^{\rho(\boldsymbol{t})}+\underline{\mathcal{R}}_{n}\left(t, t_{0}\right)
\end{aligned}
$$

(2) There exists a random variable $\xi: \Omega \rightarrow] t_{0}, t[$ so that holds:

$$
\begin{aligned}
\underline{\mathcal{R}}_{n}\left(t, t_{0}\right) & =\sum_{\substack{\boldsymbol{t} \in L T S(S) \\
\rho(\boldsymbol{t})=n+1}} \sum_{j_{1}, \ldots, j_{s(t) / 2}=1}^{m} \frac{E^{t_{0}, x_{0}}\left(F(\boldsymbol{t})\left(X_{\xi}\right)\right)}{2^{s(\boldsymbol{t}) / 2} \rho(\boldsymbol{t}) !}\left(t-t_{0}\right)^{\rho(\boldsymbol{t})} \\
& =\sum_{\substack{\boldsymbol{t} \in T S(S) \\
\rho(\boldsymbol{t})=n+1}} \sum_{j_{1}, \ldots, j_{s(t) / 2}=1}^{m} \frac{\alpha_{S}(\boldsymbol{t}) E^{t_{0}, x_{0}}\left(F(\boldsymbol{t})\left(X_{\xi}\right)\right)}{2^{s(\boldsymbol{t}) / 2} \rho(\boldsymbol{t}) !}\left(t-t_{0}\right)^{\rho(\boldsymbol{t})}
\end{aligned}
$$

Proof. The proof is very similar to the one of Theorem 3.2. Since the solution $\left(X_{t}\right)_{t \in I}$ of the autonomous Stratonovich stochastic differential equation (24) is also solution of a corresponding Itô stochastic differential equation with the 
modified drift $\tilde{a}(25)$, we refer to the corresponding Itô stochastic differential equation in the following. Then the diffusion operator $L^{0}$ results in

$$
\begin{aligned}
L^{0} & =\sum_{k=1}^{d} \tilde{a}^{k} \frac{\partial}{\partial x^{k}}+\frac{1}{2} \sum_{k, l=1}^{d} \sum_{j=1}^{m} b^{k, j} b^{l, j} \frac{\partial^{2}}{\partial x^{k} \partial x^{l}} \\
& =\sum_{k=1}^{d} \underline{a}^{k} \frac{\partial}{\partial x^{k}}+\sum_{j=1}^{m}\left(\frac{1}{\sqrt{2}} \sum_{k=1}^{d} b^{k, j} \frac{\partial}{\partial x^{k}}\right)^{2}
\end{aligned}
$$

while the operator $L^{j}$ remains as defined in (3). By reapplication of Itô's formula as performed in (4) we get

$$
E^{t_{0}, x_{0}}\left(f\left(X_{t}\right)\right)=\sum_{i=0}^{n} \frac{\left(L^{0}\right)^{i} f\left(x_{0}\right)}{i !}\left(t-t_{0}\right)^{i}+\underline{\mathcal{R}}_{n}^{*}\left(t, t_{0}\right)
$$

with $\underline{\mathcal{R}}_{n}^{*}\left(t, t_{0}\right)$ in a similar way as in (15). Thus, to prove (26) it is sufficient to show for every $n \in \mathbb{N}_{0}$ the equation

$$
\left(L^{0}\right)^{n} f\left(x_{0}\right)=\sum_{\substack{\mathbf{t} \in L T S(S) \\ \rho(\mathbf{t})=n}} \sum_{j_{1}, \ldots, j_{s(\mathbf{t}) / 2}=1}^{m} \frac{F(\mathbf{t})\left(x_{0}\right)}{2^{s(\mathbf{t}) / 2}} .
$$

The proof proceeds by induction. The case $n=0$ is trivial. Again step $n=1$ is performed for better understanding. In this case three different trees $\mathbf{t}_{1.1}=(\tau)$, $\mathbf{t}_{1.2}=\left(\sigma_{j_{1}}, \sigma_{j_{1}}\right)$ and $\mathbf{t}_{1.3}=\left(\left\{\sigma_{j_{1}}\right\}_{j_{1}}\right)$ in $\operatorname{LTS}(S)$, all of order 1 , have to be considered. For these three trees we have

$$
\begin{aligned}
L^{0}\left(F(\gamma)\left(x_{0}\right)\right) & =F\left(\mathbf{t}_{1.1}\right)\left(x_{0}\right)+\frac{1}{2} \sum_{j_{1}=1}^{m} F\left(\mathbf{t}_{1.3}\right)\left(x_{0}\right)+\frac{1}{2} \sum_{j_{1}=1}^{m} F\left(\mathbf{t}_{1.2}\right)\left(x_{0}\right) \\
& =\sum_{\substack{\mathbf{t} \in L T S(S) \\
\rho(\mathbf{t})=1}} \sum_{j_{1}, \ldots, j_{s(\mathbf{t}) / 2}=1}^{m} \frac{F(\mathbf{t})\left(x_{0}\right)}{2^{s(\mathbf{t}) / 2}} .
\end{aligned}
$$

Under the assumption that equation (30) holds for $n \in \mathbb{N}_{0}$ we proceed to prove the case $n+1$. Therefore, we get

$$
\begin{aligned}
\left(L^{0}\right)^{n+1} f\left(x_{0}\right)= & \sum_{\substack{\mathbf{t} \in L T S(S) \\
\rho(\mathbf{t})=n}} \sum_{j_{1}, \ldots, j_{s(\mathbf{t}) / 2}=1}^{m} \frac{1}{2^{s(\mathbf{t}) / 2}}\left(\sum_{k=1}^{d} \underline{a}^{k} \frac{\partial}{\partial x^{k}} F(\mathbf{t})\right. \\
& \left.+\sum_{j=1}^{m}\left(\frac{1}{\sqrt{2}} \sum_{k=1}^{d} b^{k, j} \frac{\partial}{\partial x^{k}}\right)^{2} F(\mathbf{t})\right)\left(x_{0}\right) .
\end{aligned}
$$

Firstly, we apply Lemma 2.7 to the first part of $L^{0}$ in the same way as in the proof of Theorem 3.2, however we denote for $\mathbf{u} \in \operatorname{LTS}(S)$ with $\rho(\mathbf{u})=n$ and $l(\mathbf{u})=\lambda$ the set $H_{1}(\mathbf{u})$ by $D(\mathbf{u})$ in the following. Then we proceed by considering the second part of the operator $L^{0}$, which is applied in two steps. 
Keeping Lemma 2.7 in mind, as a first step for $\mathbf{u} \in \operatorname{LTS}(S)$ with $\rho(\mathbf{u})=n$ and $l(\mathbf{u})=\lambda$, we calculate

$$
\frac{1}{\sqrt{2}} \sum_{j=1}^{m} \sum_{k=1}^{d} b^{k, j}\left(x_{0}\right) \frac{\partial}{\partial x^{k}} \sum_{j_{1}, \ldots, j_{s(\mathbf{u}) / 2}=1}^{m} \frac{F(\mathbf{u})\left(x_{0}\right)}{2^{s(\mathbf{u}) / 2}}=\sum_{\mathbf{t} \in S(\mathbf{u})} \sum_{j_{1}, \ldots, j_{s(\mathbf{u}) / 2+1}=1}^{m} \frac{F(\mathbf{t})\left(x_{0}\right)}{2^{s(\mathbf{t}) / 2}}
$$

where $S(\mathbf{u})$ denotes the set of trees $\mathbf{t} \in \operatorname{LTS}$ with $\rho(\mathbf{t})=n+\frac{1}{2},\left.\mathbf{t}^{\prime}\right|_{\{2, \ldots, \lambda\}}=\mathbf{u}^{\prime}$, $\left.\mathbf{t}^{\prime \prime}\right|_{\{1, \ldots, \lambda\}}=\mathbf{u}^{\prime \prime}$ and $\mathbf{t}^{\prime \prime}(\lambda+1)=\sigma_{j}=\sigma_{s(\mathbf{u}) / 2+1}$. Here we have $s(\mathbf{t})=s(\mathbf{u})+1$ for all $\mathbf{t} \in S(\mathbf{u})$. For the second step, we repeat the first step for $\mathbf{u}$ replaced by $\mathbf{t}=\left(\mathbf{t}^{\prime}, \mathbf{t}^{\prime \prime}\right) \in S(\mathbf{u})$.

As a result of this we get for $\mathbf{u} \in \operatorname{LTS}(S)$ with $\rho(\mathbf{u})=n$ and $l(\mathbf{u})=\lambda$

$$
\begin{aligned}
\sum_{j=1}^{m} & \left(\frac{1}{\sqrt{2}} \sum_{k=1}^{d} b^{k, j}\left(x_{0}\right) \frac{\partial}{\partial x^{k}}\right)^{2} \sum_{j_{1}, \ldots, j_{s}(\mathbf{u}) / 2}^{m} \frac{F(\mathbf{u})\left(x_{0}\right)}{2^{s(\mathbf{u}) / 2}} \\
& =\sum_{\mathbf{t}_{1} \in S(\mathbf{u})} \sum_{j_{1}, \ldots, j_{s(\mathbf{u}) / 2+1}=1}^{m} \frac{1}{\sqrt{2}} \sum_{k=1}^{d} b^{k, j_{s(\mathbf{u}) / 2+1}}\left(x_{0}\right) \frac{\partial}{\partial x^{k}} \frac{F\left(\mathbf{t}_{1}\right)\left(x_{0}\right)}{2^{s\left(\mathbf{t}_{1}\right) / 2}} \\
& =\sum_{\mathbf{t}_{1} \in S(\mathbf{u})} \sum_{\mathbf{t}_{2} \in S\left(\mathbf{t}_{1}\right)} \sum_{j_{1}, \ldots, j_{s\left(\mathbf{t}_{2}\right) / 2}=1}^{m} \frac{F\left(\mathbf{t}_{2}\right)\left(x_{0}\right)}{2^{s\left(\mathbf{t}_{2}\right) / 2}} \\
& =\sum_{\mathbf{t} \in S(S(\mathbf{u}))} \sum_{j_{1}, \ldots, j_{s(\mathbf{t}) / 2}=1}^{m} \frac{F(\mathbf{t})\left(x_{0}\right)}{2^{s(\mathbf{t}) / 2}}
\end{aligned}
$$

where $S(S(\mathbf{u}))=\bigcup_{\mathbf{t}_{1} \in S(\mathbf{u})} S\left(\mathbf{t}_{1}\right)$ is the set of all trees $\mathbf{t}=\left(\mathbf{t}^{\prime}, \mathbf{t}^{\prime \prime}\right) \in \operatorname{LTS}(S)$ with $l(\mathbf{t})=\lambda+2$ nodes, order $\rho(\mathbf{t})=n+1$, with $\left.\mathbf{t}^{\prime}\right|_{\{2, \ldots, \lambda\}}=\mathbf{u}^{\prime},\left.\mathbf{t}^{\prime \prime}\right|_{\{1, \ldots, \lambda\}}=\mathbf{u}^{\prime \prime}$ and $\mathbf{t}^{\prime \prime}(\lambda+1)=\mathbf{t}^{\prime \prime}(\lambda+2)=\sigma_{j}=\sigma_{j_{s(\mathbf{u}) / 2+1}}=\sigma_{j_{s(\mathbf{t}) / 2}}$. Again we have $s(\mathbf{t})=$ $s(\mathbf{u})+2$ for all $\mathbf{t} \in S(S(\mathbf{u}))$.

Taking together now the results for the first and the second part of $L^{0}$, the equation

$$
\left(L^{0}\right) \sum_{j_{1}, \ldots, j_{s(\mathbf{u}) / 2}=1}^{m} \frac{F(\mathbf{u})\left(x_{0}\right)}{2^{s(\mathbf{u}) / 2}}=\sum_{\mathbf{t} \in D(\mathbf{u}) \cup S(S(\mathbf{u}))} \sum_{j_{1}, \ldots, j_{s(\mathbf{t}) / 2}=1}^{m} \frac{F(\mathbf{t})\left(x_{0}\right)}{2^{s(\mathbf{t}) / 2}}
$$

holds for every $\mathbf{u} \in \operatorname{LTS}(S)$ with $\rho(\mathbf{u})=n$. Now it is easily seen that

$$
\bigcup_{\substack{\mathbf{u} \in L T S(S) \\ \rho(\mathbf{u})=n}} D(\mathbf{u}) \cup S(S(\mathbf{u}))=\{\mathbf{t} \in \operatorname{LTS}(S): \rho(\mathbf{t})=n+1\}
$$

and by applying (34) and (35) to (31) we thus arrive at (30) with $n$ replaced by $n+1$, which completes the proof of the first part of Theorem 4.2. Finally, we have to prove that $\underline{\mathcal{R}}_{n}\left(t, t_{0}\right)=\underline{\mathcal{R}}_{n}^{*}\left(t, t_{0}\right)$. This follows by similar considerations as performed in the proof of Theorem 3.2. 
A spreadsheet containing all trees $\mathbf{t}$ of the set $T S(S)$ up to order 2.0 together with the corresponding graph can be found in Table 1.

\section{Stochastic Taylor series for Itô and Stratonovich SDE systems}

Proposition 5.1 For $R_{n}\left(t, t_{0}\right)=\mathcal{R}_{n}\left(t, t_{0}\right)$ and for $R_{n}\left(t, t_{0}\right)=\underline{\mathcal{R}}_{n}\left(t, t_{0}\right)$, some $r \in \mathbb{N}$ and constants $C_{1}, C_{2}>0$ holds

$$
\left|R_{n}\left(t, t_{0}\right)\right| \leq C_{1}\left(1+\left\|x_{0}\right\|^{2 r}\right) \cdot \exp \left(C_{2}\left(t-t_{0}\right)\right) \frac{\left(t-t_{0}\right)^{n+1}}{(n+1) !}
$$

Proof. Since $f, a^{i}, \tilde{a}^{i}, b^{i, j} \in C_{P}^{2(n+1)}\left(\mathbb{R}^{d}, \mathbb{R}\right)$ for $i=1, \ldots, d$ and $j=1, \ldots, m$ we have $\left(L^{0}\right)^{n+1} f \in C_{P}\left(\mathbb{R}^{d}, \mathbb{R}\right)$. Due to the Existence and Uniqueness Theorem, for every $T>0$ there exists a constant $C$ which depends only on $T, l \in \mathbb{N}$ and the Lipschitz constant of $a, \tilde{a}$ and $b$, so that for all $t \in\left[t_{0}, T\right]$ the estimation

$$
E\left(\left\|X_{t}\right\|^{2 l}\right) \leq\left(1+E\left(\left\|x_{0}\right\|^{2 l}\right)\right) \cdot \exp \left(C\left(t-t_{0}\right)\right)
$$

holds $[1,7]$. Therefore, with (10) for some $r \in \mathbb{N}$ we obtain

$$
\begin{aligned}
\left|R_{n}\left(t, t_{0}\right)\right| & \leq \int_{t_{0}}^{t} \int_{t_{0}}^{s_{n}} \ldots \int_{t_{0}}^{s_{1}} E^{t_{0}, x_{0}}\left(\left|\left(L^{0}\right)^{n+1} f\left(X_{s}\right)\right|\right) d s d s_{1} \ldots d s_{n} \\
& \leq \int_{t_{0}}^{t} \int_{t_{0}}^{s_{n}} \ldots \int_{t_{0}}^{s_{1}} E^{t_{0}, x_{0}}\left(K_{1}\left(1+\left\|X_{s}\right\|^{2 r}\right)\right) d s d s_{1} \ldots d s_{n} \\
& \leq \frac{\left(t-t_{0}\right)^{n+1}}{(n+1) !} K_{2}\left(1+E^{t_{0}, x_{0}}\left(\left\|x_{0}\right\|^{2 r}\right)\right) \cdot \exp \left(K_{3}\left(t-t_{0}\right)\right)
\end{aligned}
$$

with some constants $K_{1}, K_{2}, K_{3}>0$.

Corollary 5.2 Let $\left(X_{t}\right)_{t \in I}$ be the solution of the stochastic differential equation (2) with initial value $X_{t_{0}}=x_{0} \in \mathbb{R}^{d}$. Then for $f, a^{i}, \tilde{a}^{i}, b^{i, j} \in C_{P}^{\infty}\left(\mathbb{R}^{d}, \mathbb{R}\right)$ for $i=1, \ldots, d$ and $j=1, \ldots, m$ and for every $t \in\left[t_{0}, T\right]$ the following expansion holds:

$$
\begin{aligned}
E^{t_{0}, x_{0}}\left(f\left(X_{t}\right)\right) & =\sum_{\boldsymbol{t} \in L T S(*)} \sum_{j_{1}, \ldots, j_{s(t) / 2}=1}^{m} \frac{F(\boldsymbol{t})\left(x_{0}\right)}{2^{s(\boldsymbol{t}) / 2} \rho(\boldsymbol{t}) !}\left(t-t_{0}\right)^{\rho(\boldsymbol{t})} \\
& =\sum_{\boldsymbol{t} \in T S(*)} \sum_{j_{1}, \ldots, j_{s(t) / 2}=1}^{m} \frac{\alpha_{*}(\boldsymbol{t}) F(\boldsymbol{t})\left(x_{0}\right)}{2^{s(\boldsymbol{t}) / 2} \rho(\boldsymbol{t}) !}\left(t-t_{0}\right)^{\rho(\boldsymbol{t})}
\end{aligned}
$$

Here, $*=I$ for the Itô version of SDE (2), and $*=S$ for the Stratonovich version of $S D E(2)$.

Proof. This follows from Theorem 3.2 in case of Itô calculus and Theorem 4.2 for Stratonovich calculus, together with Proposition 5.1. For the convergence 
of the infinite series (39) one has to prove that $\lim _{n \rightarrow \infty} \mathcal{R}_{n}\left(t, t_{0}\right)=0$ and $\lim _{n \rightarrow \infty} \underline{\mathcal{R}}_{n}\left(t, t_{0}\right)=0$, which follows from (36).

\section{Example}

Table 1 contains all S-trees of the subsets $T S(I)$ and $T S(S)$ up to order two and the corresponding cardinalities $\alpha_{I}$ and $\alpha_{S}$. The cardinalities can be determined very easily as the number of possibilities to build up the considered tree due to the corresponding rules of growth in Definition 3.1 and Definition 4.1. As an example, we consider the truncated expansion of the expectation of

\begin{tabular}{|c|c|c|c|c||c|c|c|c|c|}
\hline $\mathbf{t}$ & tree & $\alpha_{I}$ & $\alpha_{S}$ & $\rho$ & $\mathbf{t}$ & tree & $\alpha_{I}$ & $\alpha_{S}$ & $\rho$ \\
\hline \hline $\mathbf{t}_{0.1}$ & $\gamma$ & 1 & 1 & 0 & $\mathbf{t}_{2.11}$ & $\left(\sigma_{j_{1}}, \sigma_{j_{1}}, \sigma_{j_{2}}, \sigma_{j_{2}}\right)$ & 1 & 1 & 2 \\
\hline $\mathbf{t}_{1.1}$ & $(\tau)$ & 1 & 1 & 1 & $\mathbf{t}_{2.12 a}$ & $\left(\sigma_{j_{1}}, \sigma_{j_{1}},\left\{\sigma_{j_{2}}\right\}_{j_{2}}\right)$ & 0 & 2 & 2 \\
$\mathbf{t}_{1.2}$ & $\left(\sigma_{j_{1}}, \sigma_{j_{1}}\right)$ & 1 & 1 & 1 & $\mathbf{t}_{2.12 b}$ & $\left(\sigma_{j_{1}}, \sigma_{j_{2}},\left\{\sigma_{j_{2}}\right\}_{j_{1}}\right)$ & 4 & 4 & 2 \\
$\mathbf{t}_{1.3}$ & $\left(\left\{\sigma_{j_{1}}\right\}_{j_{1}}\right)$ & 0 & 1 & 1 & $\mathbf{t}_{2.13 a}$ & $\left(\sigma_{j_{1}},\left\{\sigma_{j_{2}}, \sigma_{j_{2}}\right\}_{j_{1}}\right)$ & 2 & 2 & 2 \\
\hline $\mathbf{t}_{2.1}$ & $([\tau])$ & 1 & 1 & 2 & $\mathbf{t}_{2.13 b}$ & $\left(\sigma_{j_{2}},\left\{\sigma_{j_{2}}, \sigma_{j_{1}}\right\}_{j_{1}}\right)$ & 0 & 2 & 2 \\
$\mathbf{t}_{2.2}$ & $(\tau, \tau)$ & 1 & 1 & 2 & $\mathbf{t}_{2.14 a}$ & $\left(\sigma_{j_{1}},\left\{\left\{\sigma_{j_{2}}\right\}_{j_{2}}\right\}_{j_{1}}\right)$ & 0 & 2 & 2 \\
$\mathbf{t}_{2.3}$ & $\left(\left[\left\{\sigma_{j_{1}}\right\}_{j_{1}}\right]\right)$ & 0 & 1 & 2 & $\mathbf{t}_{2.14 b}$ & $\left(\sigma_{j_{2}},\left\{\left\{\sigma_{j_{2}}\right\}_{j_{1}}\right\}_{j_{1}}\right)$ & 0 & 2 & 2 \\
$\mathbf{t}_{2.4}$ & $\left(\left[\sigma_{j_{1}}, \sigma_{j_{1}}\right]\right)$ & 1 & 1 & 2 & $\mathbf{t}_{2.15 a}$ & $\left(\left\{\sigma_{j_{1}}\right\}_{j_{1}},\left\{\sigma_{j_{2}}\right\}_{j_{2}}\right)$ & 0 & 1 & 2 \\
$\mathbf{t}_{2.5}$ & $\left(\sigma_{j_{1}},\left[\sigma_{j_{1}}\right]\right)$ & 2 & 2 & 2 & $\mathbf{t}_{2.15 b}$ & $\left(\left\{\sigma_{j_{2}}\right\}_{j_{1}},\left\{\sigma_{j_{2}}\right\}_{j_{1}}\right)$ & 2 & 2 & 2 \\
$\mathbf{t}_{2.6}$ & $\left(\left\{\sigma_{j_{1}}\right\}_{j_{1}}, \tau\right)$ & 0 & 2 & 2 & $\mathbf{t}_{2.16}$ & $\left(\left\{\sigma_{j_{1}}, \sigma_{j_{2}}, \sigma_{j_{2}}\right\}_{j_{1}}\right)$ & 0 & 1 & 2 \\
$\mathbf{t}_{2.7}$ & $\left(\sigma_{j_{1}}, \sigma_{j_{1}}, \tau\right)$ & 2 & 2 & 2 & $\mathbf{t}_{2.17 a}$ & $\left(\left\{\sigma_{j_{1}},\left\{\sigma_{j_{2}}\right\}_{j_{2}}\right\}_{j_{1}}\right)$ & 0 & 1 & 2 \\
$\mathbf{t}_{2.8}$ & $\left(\sigma_{j_{1}},\{\tau\}_{j_{1}}\right)$ & 2 & 2 & 2 & $\mathbf{t}_{2.17 b}$ & $\left(\left\{\sigma_{j_{2}},\left\{\sigma_{j_{2}}\right\}_{j_{1}}\right\}_{j_{1}}\right)$ & 0 & 2 & 2 \\
$\mathbf{t}_{2.9}$ & $\left(\left\{\{\tau\}_{j_{1}}\right\}_{j_{1}}\right)$ & 0 & 1 & 2 & $\mathbf{t}_{2.18}$ & $\left(\left\{\left\{\sigma_{j_{2}}, \sigma_{j_{2}}\right\}_{j_{1}}\right\}_{j_{1}}\right)$ & 0 & 1 & 2 \\
$\mathbf{t}_{2.10}$ & $\left.\left(\left\{\sigma_{j_{1}}, \tau\right\}\right\}_{j_{1}}\right)$ & 0 & 1 & 2 & $\mathbf{t}_{2.19}$ & $\left(\left\{\left\{\left\{\sigma_{j_{2}}\right\}_{j_{2}}\right\}_{j_{1}}\right\}_{j_{1}}\right)$ & 0 & 1 & 2 \\
\hline
\end{tabular}

Table 1

Trees $t \in T S$ for the Taylor expansion of the solution of a SDE w.r.t. either Itô or Stratonovich calculus of order 0,1 and 2 .

some functional $f$ of the solution $X_{t}$ of the Itô SDE (11). Then, the expansion 
up to order two is given by

$$
\begin{aligned}
E^{t_{0}, x_{0}}\left(f\left(X_{t}\right)\right)= & F\left(\mathbf{t}_{0.1}\right)\left(x_{0}\right)+F\left(\mathbf{t}_{1.1}\right)\left(x_{0}\right) \cdot\left(t-t_{0}\right)+\sum_{j_{1}=1}^{m} \frac{F\left(\mathbf{t}_{1.2}\right)\left(x_{0}\right)}{2}\left(t-t_{0}\right) \\
& +\frac{F\left(\mathbf{t}_{2.1}\right)\left(x_{0}\right)}{2}\left(t-t_{0}\right)^{2}+\frac{F\left(\mathbf{t}_{2.2}\right)\left(x_{0}\right)}{2}\left(t-t_{0}\right)^{2} \\
& +\sum_{j_{1}=1}^{m} \frac{F\left(\mathbf{t}_{2.4}\right)\left(x_{0}\right)}{4}\left(t-t_{0}\right)^{2}+\sum_{j_{1}=1}^{m} \frac{F\left(\mathbf{t}_{2.5}\right)\left(x_{0}\right)}{2}\left(t-t_{0}\right)^{2} \\
& +\sum_{j_{1}=1}^{m} \frac{F\left(\mathbf{t}_{2.7}\right)\left(x_{0}\right)}{2}\left(t-t_{0}\right)^{2}+\sum_{j_{1}=1}^{m} \frac{F\left(\mathbf{t}_{2.8}\right)\left(x_{0}\right)}{2}\left(t-t_{0}\right)^{2} \\
& +\sum_{j_{1}, j_{2}=1}^{m} \frac{F\left(\mathbf{t}_{2.11}\right)\left(x_{0}\right)}{8}\left(t-t_{0}\right)^{2}+\sum_{j_{1}, j_{2}=1}^{m} \frac{F\left(\mathbf{t}_{2.12 b}\right)\left(x_{0}\right)}{2}\left(t-t_{0}\right)^{2} \\
& +\sum_{j_{1}, j_{2}=1}^{m} \frac{F\left(\mathbf{t}_{2.13 a}\right)\left(x_{0}\right)}{4}\left(t-t_{0}\right)^{2}+\sum_{j_{1}, j_{2}=1}^{m} \frac{F\left(\mathbf{t}_{2.15 b}\right)\left(x_{0}\right)}{4}\left(t-t_{0}\right)^{2} \\
& +\mathcal{R}_{2}\left(t, t_{0}\right) .
\end{aligned}
$$

Finally, we calculate the elementary differentials for the trees appearing in the truncated expansion (40). As a result of this, we get

$$
\begin{aligned}
E^{t_{0}, x_{0}}( & \left.f\left(X_{t}\right)\right)=f\left(x_{0}\right)+\sum_{J=1}^{d} \frac{\partial f\left(x_{0}\right)}{\partial x^{J}} a^{J}\left(x_{0}\right) \cdot\left(t-t_{0}\right) \\
& +\frac{1}{2} \sum_{j_{1}=1}^{m} \sum_{J, K=1}^{d} \frac{\partial^{2} f\left(x_{0}\right)}{\partial x^{J} \partial x^{K}} b^{J, j_{1}}\left(x_{0}\right) b^{K, j_{1}}\left(x_{0}\right) \cdot\left(t-t_{0}\right) \\
& +\frac{1}{2} \sum_{J, K=1}^{d} \frac{\partial f\left(x_{0}\right)}{\partial x^{J}} \frac{\partial a^{J}\left(x_{0}\right)}{\partial x^{K}} a^{K}\left(x_{0}\right) \cdot\left(t-t_{0}\right)^{2} \\
& +\frac{1}{2} \sum_{J, K=1}^{d} \frac{\partial^{2} f\left(x_{0}\right)}{\partial x^{J} \partial x^{K}} a^{J}\left(x_{0}\right) a^{K}\left(x_{0}\right) \cdot\left(t-t_{0}\right)^{2} \\
& +\frac{1}{4} \sum_{j_{1}=1}^{m} \sum_{J, K, L=1}^{d} \frac{\partial f\left(x_{0}\right)}{\partial x^{J}} \frac{\partial^{2} a^{J}\left(x_{0}\right)}{\partial x^{K} \partial x^{L}} b^{K, j_{1}}\left(x_{0}\right) b^{L, j_{1}}\left(x_{0}\right) \cdot\left(t-t_{0}\right)^{2} \\
& +\frac{1}{2} \sum_{j_{1}=1}^{m} \sum_{J, K, L=1}^{d} \frac{\partial^{2} f\left(x_{0}\right)}{\partial x^{J} \partial x^{K}} b^{J, j_{1}}\left(x_{0}\right) \frac{\partial a^{K}\left(x_{0}\right)}{\partial x^{L}} b^{L, j_{1}}\left(x_{0}\right) \cdot\left(t-t_{0}\right)^{2} \\
& +\frac{1}{2} \sum_{j_{1}=1}^{m} \sum_{J, K, L=1}^{d} \frac{\partial^{3} f\left(x_{0}\right)}{\partial x^{J} \partial x^{K} \partial x^{L}} b^{J, j_{1}}\left(x_{0}\right) b^{K, j_{1}}\left(x_{0}\right) a^{L}\left(x_{0}\right) \cdot\left(t-t_{0}\right)^{2} \\
& +\frac{1}{2} \sum_{j_{1}=1}^{m} \sum_{J, K, L=1}^{d} \frac{\partial^{2} f\left(x_{0}\right)}{\partial x^{J} \partial x^{K}} b^{J, j_{1}}\left(x_{0}\right) \frac{\partial b^{K, j_{1}}\left(x_{0}\right)}{\partial x^{L}} a^{L}\left(x_{0}\right) \cdot\left(t-t_{0}\right)^{2}
\end{aligned}
$$




$$
\begin{aligned}
+ & \frac{1}{8} \sum_{j_{1}, j_{2}=1}^{m} \sum_{J, K, L, M=1}^{d} \frac{\partial^{4} f\left(x_{0}\right)}{\partial x^{J} \partial x^{K} \partial x^{L} \partial x^{M}} b^{J, j_{1}}\left(x_{0}\right) b^{K, j_{1}}\left(x_{0}\right) \times \\
& \times b^{L, j_{2}}\left(x_{0}\right) b^{M, j_{2}}\left(x_{0}\right) \cdot\left(t-t_{0}\right)^{2} \\
+ & \frac{1}{2} \sum_{j_{1}, j_{2}=1}^{m} \sum_{J, K, L, M=1}^{d} \frac{\partial^{3} f\left(x_{0}\right)}{\partial x^{J} \partial x^{K} \partial x^{L}} b^{J, j_{1}}\left(x_{0}\right) b^{K, j_{2}}\left(x_{0}\right) \times \\
& \times \frac{\partial b^{L, j_{1}}\left(x_{0}\right)}{\partial x^{M}} b^{M, j_{2}}\left(x_{0}\right) \cdot\left(t-t_{0}\right)^{2} \\
+ & \frac{1}{4} \sum_{j_{1}, j_{2}=1}^{m} \sum_{J, K, L, M=1}^{d} \frac{\partial^{2} f\left(x_{0}\right)}{\partial x^{J} \partial x^{K}} b^{J, j_{1}}\left(x_{0}\right) \frac{\partial^{2} b^{K, j_{1}}\left(x_{0}\right)}{\partial x^{L} \partial x^{M}} \times \\
& \times b^{L, j_{2}}\left(x_{0}\right) b^{M, j_{2}}\left(x_{0}\right) \cdot\left(t-t_{0}\right)^{2} \\
+ & \frac{1}{4} \sum_{j_{1}, j_{2}=1}^{m} \sum_{J, K, L, M=1}^{d} \frac{\partial^{2} f\left(x_{0}\right)}{\partial x^{J} \partial x^{K}} \frac{\partial b^{J, j_{1}}\left(x_{0}\right)}{\partial x^{L}} b^{L, j_{2}}\left(x_{0}\right) \times \\
& \times \frac{\partial b^{K, j_{1}}\left(x_{0}\right)}{\partial x^{M}} b^{M, j_{2}}\left(x_{0}\right) \cdot\left(t-t_{0}\right)^{2}+\mathcal{R}_{2}\left(t, t_{0}\right) .
\end{aligned}
$$

Remark 6.1 We have to point out that Theorem 3.2 as well as Theorem 4.2 provide an expansion of functionals of the solution of the corresponding deterministic differential equation system, i.e. SDE (2) with $b \equiv 0$. Here, trees composed of the root $\gamma$ and deterministic nodes $\tau$ have to be considered only. Further, the presented Taylor formulas coincide with the ones used for the numerical analysis of deterministic Runge-Kutta methods (see, e.g. [4]).

To illustrate the stochastic Taylor expansion, we consider the stochastic differential equation

$$
d X_{t}=\alpha X_{t} d t+\beta X_{t} d W_{t}, \quad X_{0}=x_{0} \in \mathbb{R}
$$

in the case of $d=m=1$, i.e. for a one-dimensional Wiener process, with some constants $\alpha, \beta \in \mathbb{R}$. If we choose $f(x)=x$, the expectation of the solution can be calculated as $E^{0, x_{0}}\left(X_{t}\right)=x_{0} \exp (\alpha t)$. In the case of $m=1$, the single index $j_{1}$ at the trees can be omitted since we have $j_{1} \equiv 1$. By the determination of the elementary differentials appearing in (40) we yield the following truncated expansion

$$
E^{0, x_{0}}\left(X_{t}\right)=x_{0}+\alpha x_{0}(t-0)+\frac{1}{2} \alpha^{2} x_{0}(t-0)^{2}+\mathcal{R}_{2}(t, 0)
$$

which coincides with the order two Taylor polynomial of the exact solution.

The Taylor expansion can also be calculated with the aid of hirarchical sets and the operators $L^{0}$ and $L^{j}$ introduced by Platen and Wagner (see $[8,10]$ ). 
However, the rooted tree theory allows the direct determination of each elementary differential by the corresponding tree which makes it more transparent and allows an easy analysis of the structure of the elementary differentials. For example, the rooted tree approach serves as an appropriate tool for the determination of stochastic Runge-Kutta methods (see [2,11]).

\section{References}

[1] L. Arnold, Stochastic differential equations (John Wiley \& Sons, New York, 1974).

[2] K. Burrage and P. M. Burrage, Order conditions of stochastic Runge-Kutta methods by B-series, SIAM J. Numer. Anal., 38, No. 5, (2000) 1626-1646.

[3] K. Burrage and P. M. Burrage, High strong order explicit Runge-Kutta methods for stochastic ordinary differential equations, Appl. Numer. Math., 22, No. 1-3, (1996) 81-101.

[4] J. C. Butcher, The numerical analysis of ordinary differential equations: RungeKutta and general linear methods (John Wiley \& Sons, Chichester, 1987).

[5] E. B. Dynkin, Markov Processes (Springer-Verlag, Berlin, Vol. I,II, 1965).

[6] N. Ikeda and S. Watanabe, Stochastic Differential Equations and Diffusion Processes (North-Holland, Amsterdam (Kodansha Ltd., Tokyo), 1989).

[7] I. Karatzas and S. E. Shreve, Brownian Motion and Stochastic Calculus (Springer New-York, Berlin, 1999).

[8] P. E. Kloeden and E. Platen, Numerical Solution of Stochastic Differential Equations (Applications of Mathematics 23, Springer-Verlag, Berlin, 1999).

[9] B. Øksendal, Stochastic Differential Equations (Applications of Mathematics 21, Springer-Verlag, Berlin, 1998).

[10] E. Platen and W. Wagner, On a Taylor formula for a class of Itô processes, Probab. Math. Statist. 3 (1982) 37-51.

[11] A. Rößler, Runge-Kutta Methods for the Numerical Solution of Stochastic Differential Equations, Ph.D. thesis, Darmstadt University of Technology. (Shaker Verlag, Aachen, 2003).

[12] D. W. Stroock and S. R. S. Varadhan, Multidimensional Diffusion Processes (Springer-Verlag Berlin, 1979). 